\title{
A re-audit of the management of diabetic ketoacidosis after the introduction of a local protocol based on the JBDS guidelines: then and now
}

\author{
HIBBA KURDI, LEO P PINTO, FIONA J SMEETON
}

\begin{abstract}
Background: In 2010 the Joint British Diabetes Societies Inpatient Care Group issued guidelines for the management of Diabetic Ketoacidosis. A new management guideline and bedside chart based on these guidelines were introduced in our Health Board in 2012.

Methods: A retrospective re-audit of 33 patients managed according to the new guideline, admitted from October 2013 to October 2014. Results were compared to 2010 prior to implementation of the guideline and the first audit cycle September 2012 to 2013 immediately after.

Results: $\mathbf{3}$ admissions a month. Median age was 34 years, $94 \%$ admitted with established DKA and vomiting $(70 \%)$, as the commonest presenting symptom. Length of stay: median of 2 days, with mortality of $0 \%$. Median time to IV fluids: 35 (2 - 420) minutes, time to fixed rate intravenous insulin infusion: 40 ( 2 to 210) minutes. Long acting insulin continued in $94 \%$ with $21 \%$ hypoglycaemia $(n=7)$ and $6 \%$ hypokalaemia rates $(n=2)$. Median time to resolution of ketosis: 12 hours. Variable rate insulin started in $55 \%$ post DKA protocol, due to ongoing nausea and vomiting. $100 \%$ of patients were reviewed by the inpatient diabetes care team.

Conclusions: Sustained improvements in median times to insulin, fluids and $>\mathbf{5 0} \%$ reduction in hypoglycaemia. Reduced time on intravenous insulin compared to pre guideline in $\mathbf{2 0 1 0}$ with an objective end point for resolution of ketoacidosis. Bedside potassium monitoring remains suboptimal.

Br J Diabetes 2017;17:52-57
\end{abstract}

Key words: diabetic ketoacidosis, inpatient care, diabetes mellitus, Joint British Diabetes Society, audit

Nevill Hall Hospital, Aneurin Bevan University Health Board, South Wales, UK

Address for correspondence: Dr Fiona J Smeeton

Department of Diabetes and Endocrinology, Nevill Hall Hospital, Aneurin

Bevan University Health Board, Brecon Rd, Abergavenny, South Wales,

NP7 7EG, UK

Tel: $+44(0) 1873732732$

E-mail: fiona.smeeton@wales.nhs.uk

http://dx.doi.org/10.15277/bjd.2017.133

\section{Introduction}

The management of diabetic ketoacidosis (DKA) has been a topic of conversation amongst physicians since it was first alluded to in 1886 by a German pathologist named Julius Dreschfeld. ${ }^{1}$ The condition remained fatal until the discovery of insulin in the 1920 s; mortality rates are still reported to be between $0.7 \%$ and $5 \% .^{2}$

In 2010 the Joint British Diabetes Societies Inpatient Care Group (JBDS IP Group) published a guideline for the management of DKA which was revised in 2013. ${ }^{3}$ This document formed the foundation of Aneurin Bevan University Health Board's (ABUHB) own guidelines and protocol which came into effect from 10 September 2012.

The local guideline and accompanying bedside treatment chart were introduced along with staff education and publicity highlighting the key changes in the management of DKA. The bedside chart provides a stepwise management approach and prompts for appropriate treatment and monitoring in accordance with the JBDS IP Group guidelines. A baseline audit of the management of DKA prior to the implementation of the new chart and guideline was completed in 2010 .

A retrospective audit of adult patients with DKA was undertaken after introduction of the protocol between 10 September 2012 and 10 September 2013. Following this audit, the results were presented and further education provided.

Opportunities to further improve the management of DKA were highlighted in the form of lectures at grand rounds and audit meetings; medical teaching focused on the admitting areas in the hospital as well as sessions for nursing staff and with support from the pathology point-of-care team. Following this period of consolidation, the audit was then repeated between October 2013 and October 2014 to assess progress made.

In this document the first audit 2012-2013, immediately after introduction of the local protocol, is referred to as cycle 1 and the re-audit cycle 2013-2014 is referred to as cycle 2. We present completed data from cycle 2 here. Where possible, a comparison between cycles 1 and 2 is made. Data from these two cycles are then compared with the baseline audit in 2010.

\section{Objectives}

The aim was to audit the management of DKA in a 420-bed district general hospital after the introduction of a new bedside chart 
Table 1 Comparison of results across audit cycles 1 and 2

$\begin{array}{ll}2013 / 2014 & 2012 / 2013 \\ \text { (audit cycle 2 after } & \text { (audit cycle 1 after } \\ \text { local DKA protocol) } & \text { local DKA protocol) }\end{array}$

\begin{tabular}{|c|c|c|}
\hline \multicolumn{3}{|l|}{ Demographics } \\
\hline $\begin{array}{l}\text { Total number of } \\
\text { patients included } \\
\text { per audit cycle }\end{array}$ & 33 & 33 \\
\hline *Age (years) & 34 & 36 \\
\hline *Admissions per month & 3 & 3 \\
\hline *Length of stay (days) & 2 & 3 \\
\hline Mortality (\%) & 0 & 9 \\
\hline \multicolumn{3}{|l|}{ Diagnosis } \\
\hline $\begin{array}{l}\text { Commonest presenting } \\
\text { complaint }\end{array}$ & Vomiting & Vomiting \\
\hline $\begin{array}{l}\text { Commonest DKA } \\
\text { precipitant }\end{array}$ & Infection & Infection \\
\hline
\end{tabular}

\begin{tabular}{|c|c|c|c|}
\hline \multicolumn{3}{|c|}{ Management; within 1 hour of DKA protocol start (\%) } & publication of the JBDS quidelines: \\
\hline IV fluids & 76 & 70 & \multirow{2}{*}{$\begin{array}{l}\text { 1. Glucose } \geq 11 \mathrm{mmol} / \mathrm{L} \text { and } \mathrm{a} \mathrm{pH} \text { of } \leq 7.3 \text { and urinary ketones } \\
\geq 2 \text {. }\end{array}$} \\
\hline FRIII & 73 & 55 & \\
\hline Manage & & & 2. Patients aged $\geq 18$ years. \\
\hline
\end{tabular}

Capillary blood glucose 100

(measured per protocol)

Potassium (measured

per protocol)

Ketones (measured

per protocol)

Long-acting insulin

continued

Resolution of ketosis

in 24 hours

Use of VRIII

52

93

67

10090

$94 \quad 43$

$97 \quad 97$

$55 \quad 57$

\section{Complications (\%)}

Hypoglycaemia

Hypokalaemia

2

6

10

27

\section{Other (\%)}

Inpatient diabetes

team review

Thromboprophylaxis

Outpatient review

30 days after discharge

$100 \quad 100$

$85 \quad 94$

$60 \quad 97$

*All figures are given as median unless otherwise stated.

DKA, diabetic ketoacidosis; FRIII, fixed rate intravenous insulin infusion; VRIII, variable rate intravenous insulin infusion.

and guideline implementing the recommended changes from the JBDS IP Group. Nevill Hall Hospital (NHH) is a district general hospital and is part of ABUHB in South Wales. We wanted to assess both improvements in the management and time to resolution of DKA and also assess if there were any adverse events such as hypokalaemia or hypoglycaemia associated with the use of fixed rate intravenous insulin infusion (FRIII).
Where possible, outcome end points will be compared against the national average, as stated in the national survey by Dhatariya et al in 2014, ${ }^{4}$ and against the audit data from both 2012 immediately after the new guidelines and chart and 2010 prior to the introduction of the local protocol. A summary of results across audit cycles 1 and 2 can be found in Table 1. The baseline audit in 2010 will be discussed separately.

\section{Criteria}

The following audit criteria were adhered to throughout audit cycles 1 and 2:

A diagnosis of DKA where the following requirements were met:

1. Capillary blood glucose $\geq 11 \mathrm{mmol} / \mathrm{L}$ and capillary ketones $\geq 3$ $\mathrm{mmol} / \mathrm{L}$ (or severe ketonuria $\geq 2$ ) and venous $\mathrm{pH} \leq 7.3$ and/or bicarbonate $\leq 15 \mathrm{mmol} / \mathrm{L}$, based on the JBDS DKA guidelines. ${ }^{3}$

2. Patients aged $\geq 18$ years.

The baseline audit in 2010 used local criteria based on variable rate intravenous insulin infusion (VRIII) to diagnose DKA before publication of the JBDS guidelines:

$\geq 2$.
Patients aged $\geq 18$ years.

\section{Methods}

A search was conducted on ABUHB's patient database system. This allowed for easy identification of discharge notifications where the diagnosis entered was that of DKA. Principles of information governance were adhered to at all times.

The results of the above search were then cross-referenced against the paper database kept by the diabetes specialist nurses for completion and notes analysed retrospectively against the recommended standards.

\section{Data collection}

Relevant data were transcribed onto an audit data capture sheet modelled on the NICE audit tool and Excel spread sheet designed specifically for audit purposes.

All biochemical samples were analysed in $\mathrm{NHH}^{\prime}$ s onsite laboratory as per standard.

\section{Results}

Demographics

A total of 47 discharge summaries were identified as a result of the initial search; 33 patients, all of Caucasian descent, were found to have a correct diagnosis of DKA and were therefore included in subsequent data analysis. Patients with an incorrect diagnosis of DKA $(n=9)$ and those with inaccessible patient notes were excluded from the audit $(n=5)$.

The median age was 34 years with a relatively equal spread of male $(48 \% ; n=16)$ and female patients $(52 \% ; n=17)$. There was an average of three admissions per month (October 2013 to October 2014). This is the same as audit cycle 1 (September 2012 to September 2013), with peaks seen in December, March and August. One patient was readmitted with a further episode of DKA within the audit period. 
Thirty-one patients (94\%) were admitted with an established episode of DKA. Two patients developed DKA during their inpatient stay. There were no deaths in the study population in 2014 but mortality was $9 \%(n=3)$ in the previous year (Table 1). Two deaths occurred from sepsis despite adequate treatment of the DKA and one was due to a delay in establishing intravenous access and therefore initiating FRIII. Thirty patients had an established diagnosis of type 1 diabetes, two patients were newly diagnosed after their presentation with DKA and one patient had a prior diagnosis of type 2 diabetes treated with insulin.

The median length of stay was 2 days, which is slightly better than the national average which was a median of 2.6 days in $2014 .{ }^{3}$

\section{Diagnosis}

The most common presenting symptoms of DKA were vomiting and abdominal pain. Symptoms not falling into these categories were labelled as 'other' or otherwise as infrequent. Vomiting was recorded in $70 \%$ as the initial presenting complaint and abdominal pain in $9 \%(n=3)$. This was similar in both audits.

Infection was the most frequent recorded precipitant totalling $33 \%(n=11$; Table 1). The DKA guideline coincided with the introduction of the Sepsis Six pathway throughout $A B U H B$, leading to improvements in the recognition and treatment of sepsis. Alcohol was also an important precipitant throughout all audit cycles and is often overlooked in the initial assessment of DKA.

The documentation of admission blood ketones presents a challenge due to compatibility, with the blood ketone records in A\&E not appearing on the hospital pathology record. Those taken on the wards are accessible on the pathology results system. These results were accessed if recorded in the case notes.

\section{Management}

\section{DKA protocol recommendations: management within 1} hour of diagnosis

Twenty-five patients (76\%) received intravenous (IV) fluids within 1 hour of admission, as recommended by the protocol (range 2-420 min). This is an improvement from the $70 \%$ of cycle 1 immediately after introduction of the local protocol. Fourteen $(42 \%)$ of these received fluids within $30 \mathrm{~min}$. The median time from diagnosis to starting IV fluids was $35 \mathrm{~min}$. All DKA admissions were treated with $0.9 \%$ normal saline. Delays in giving fluids were documented as being due to difficult intravenous access.

FRIII was used in all cases. Twenty-four DKA admissions (73\%) had FRIII started within 1 hour of admission (range 2-210 min); 16 of these (49\%) occurred within 30 min of admission. This is an improvement from audit cycle 1 when $55 \%$ received IV insulin within 1 hour. Overall, the median time from diagnosis to receiving IV insulin was $40 \mathrm{~min}$.

\section{Implementation of the new guideline recommendations and complications}

The DKA protocol was used and adhered to in $97 \%$ of cases $(n=32)$. Blood ketones, glucose levels (CBG) and potassium were measured according to protocol $>90 \%$ of the time (Table 1).

Long-acting insulin was continued appropriately in $94 \%$ of cases $(n=31)$, which is a significant improvement from audit cycle 1 and highlights improvement after education reinforcing the change in practice. The continuation of long-acting insulin had not previously been a recommendation prior to the JBDS IP Group guideline and hence, in 2010, was only continued in one patient.

There were seven $(21 \%)$ recorded episodes of hypoglycaemia, which is an increase from the audit cycle $1(10 \%)$ but not worse than the national average of $26.7 \%$ reported by Dhatariya et al. ${ }^{4}$

Potassium should be measured and replaced within at least the second hour of admission. Seventeen patients (51\%) received potassium replacement within 2 hours of admission (range 2-480 $\mathrm{min}$ ). This is an improvement compared with audit cycle 1 where $36 \%$ of patients received potassium within 2 hours of admission. Despite this, cases of hypokalaemia remained low throughout audit cycle 2 (Table 1) and improved from audit cycle 1 immediately after introduction of the local protocol. This is also better than the national average for hypokalaemia (67\%) noted at 24 hours of DKA treatment. ${ }^{4}$

Ketosis had resolved by 24 hours in $>90 \%$ of cases across both audit cycles (Table 1). Median time to resolution of ketosis was 12 hours (mean 12.2 hours) compared with a median of 11 hours (mean 11.1 hours) in audit cycle 1 . The range of time on FRIII was 3-48 hours (48 hours being one outlier requiring prolonged time on the DKA protocol due to severe sepsis of unknown origin). Prior to the use of FRIII, the mean time on variable rate VRIII was 35 hours (range 5-140 hours) in 2010.

Transfer from FRIII to VRIII at resolution of ketoacidosis occurred in 55\%, which in all cases was due to continued nausea or vomiting and hence a delay in the return to usual eating. The remaining patients were transferred directly from FRIII to subcutaneous insulin with the next meal. The majority had subcutaneous insulin recommenced within 48 hours ( $n=32,97 \%)$, with $55 \%$ of those started on subcutaneous insulin within 24 hours $(n=18)$.

\section{Further aspects of inpatient care}

All of the patients were seen by the inpatient diabetes team or diabetes specialist nurse during admission. At present we are not able to provide a seven-day service for review of patients presenting with DKA by a member of the diabetes team. Therefore, those presenting out of hours were not discharged until a review by the inpatient team had taken place. Thromboprophylaxis was achieved in $85 \%$ of cases (Table 1) and all patients had an electronic discharge summary completed.

Formal outpatient clinic follow-up was achieved within 30 days for $60 \%(n=20)$; two patients $(6 \%)$ had no fixed abode and were lost to follow-up. Fifteen patients (46\%) were seen by a dietitian.

\section{Comparison of current data and 2010 audit: then and now}

There are limitations to drawing a direct comparison between the baseline audit in 2010 prior to the JBDS IP Group guidelines and 
Figure 1. Precipitating factors

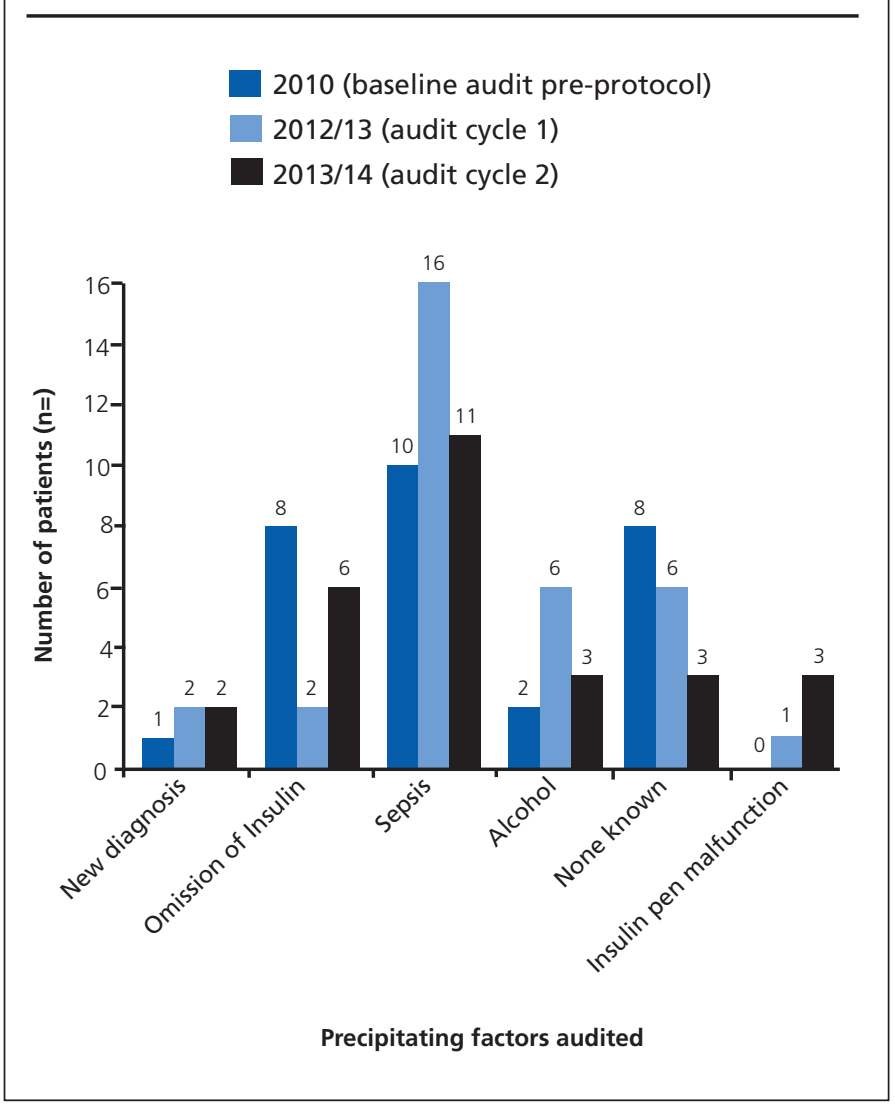

the subsequent audit cycles. Prior to the introduction of pointof-care testing for capillary blood ketones, resolution of DKA depended on the surrogate marker of capillary blood glucose, resolution of acidosis and urinary ketone testing. Intravenous insulin infusions were often continued for long periods of time without a defined end point.

The population represented among the three audit cycles is similar with an average age of 38-43 years. Median length of stay in 2010 was reported as 3 days. An increase in admissions to ITU with severe DKA is noted (20\% in 2010 vs. $36 \%$ in 2013/2014).

The most prevalent presenting symptom was vomiting, and this is unchanged throughout all of the audit cycles. This is also true for infection as the trigger for episodes of DKA. Figure 1 demonstrates the prevalence of precipitating factors across all audit cycles. It is a concern that $6 \%$ of patients $(n=2)$ developed DKA in hospital. This is similar to the higher reported values in the National DKA survey of $7.8 \%$ developing DKA during their inpatient stay. ${ }^{4}$ In the National Diabetes Inpatient Audit (NaDIA) $2016,4.4 \%$ of patients with type I diabetes developed DKA during their hospital stay. ${ }^{5}$ These events of in-hospital DKA are reported as serious incidents and work is ongoing to reduce the risk of insulin omission and the appropriate management of hyperglycaemia in hospital.

The recommendation that basal subcutaneous insulin should be continued has resulted in a change in practice from just $2 \%$
Figure 2. Percentage achieving audit standards

Audit cycle 1; 2012-2013

Reaudit cycle 2; 2013-2014

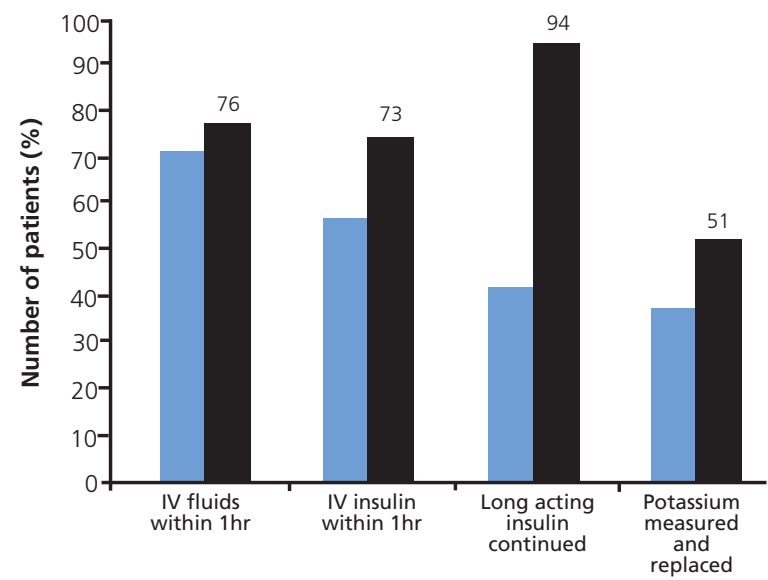

Audit standards

achieving this in 2010 to $94 \%$ during audit cycle 2 . Hypoglycaemia rates have improved with FRIII being supported by a $10 \%$ glucose infusion once the capillary blood glucose drops below $14 \mathrm{mmol} / \mathrm{L}$. The time taken from admission to starting IV insulin treatment was a median of 2.5 hours in 2010 and is currently $40 \mathrm{~min}$

Intravenous insulin infusions were continued for an average of 35 hours per patient in the 2010 audit compared with a mean of 11 hours currently, with a defined end point for resolution of ketosis. The prolonged time on IV insulin may also have contributed to the previously higher hypoglycaemia rate of $47 \%$ in 2010 on VRIII compared with our latest re-audit figure in cycle 2 of $21 \%$.

There has been an improvement in the rate of potassium replacement since the introduction of the bedside chart prompting monitoring and supplementation. This was 50\% in 2010 compared with $100 \%(n=33)$ of patients in the current audit (audit cycle 2), although only $51 \%$ of those received the potassium replacement within the recommended time as per protocol. Hypokalaemia was reported in a small number of cases and did not result in harm. Unfortunately there are no data on the rates of hypokalaemia from the 2010 audit so a direct comparison cannot be made.

There remains a degree of difficulty in potassium replacement given the risk of cardiotoxicity if not administered safely and at the correct rate. This was also identified as an area of controversy requiring further consideration in the National Survey published in $2014 .^{4}$

The proactive diabetes inpatient care team review has improved the rate of inpatient review by a specialist diabetes health professional from $83 \%$ to $100 \%$. 


\section{Discussion}

In the 2016 NaDIA, those with diabetes occupied $17 \%$ of beds in hospital across England and Wales. ${ }^{5}$ DKA has been reported to be the reason for admission in $15 \%$ of those admitted with either type 1 or type 2 diabetes mellitus. ${ }^{6}$ NaDIA 2015 reported that, amongst those with type 1 diabetes mellitus, DKA makes up $56 \%$ of the causes for hospital admission. ${ }^{6}$ This highlights the importance of the ongoing evaluation of the prevention and management of DKA despite overall improvements in mortality.

Dhatariya et al comment that it is difficult to know whether there has been an overall improvement in standards of care since hospitals adopted the JBDS DKA guidelines locally, ${ }^{4}$ as there was a lack of large-scale national audits on the management of DKA prior to this. Although our audit population is small by comparison with other audits including the national DKA survey, we have shown improvement in certain aspects of care. This is due to the combination of the introduction of the DKA guideline and bedside treatment chart, point-of-care capillary blood ketone testing and the diabetes inpatient care team (Figure 2).

The Diabetes Inpatient Care Team at $\mathrm{NHH}$ was established in June 2012, providing proactive review to hospital inpatients with diabetes and enabling early identification and management of patients admitted with DKA. This facilitated early review and timely education to both the person admitted with DKA and staff managing patients with DKA.

In addition, bedside ketone meters that detect the predominant ketone body 3-beta hydroxybutyrate were introduced into designated areas at $\mathrm{NHH}$, including $\mathrm{A} \& \mathrm{E}, \mathrm{EAU}, \mathrm{ICU}$ and the diabetes ward. This has allowed for treatment decisions to be accurately based on the suppression of ketogenesis rather than hyperglycaemia.

This audit cycle has demonstrated a clinically significant reduction of total time on intravenous insulin and an associated reduction of more than $50 \%$ in hypoglycaemic events. Overall, there has been an improvement in initiation of intravenous fluids at initial diagnosis, potassium replacement and a greater than $90 \%$ improvement in the continuation of long-acting subcutaneous insulin.

The 2013 TOPDOC study identified that just $43 \%$ of junior doctors felt 'fully confident' in managing DKA. ${ }^{7}$ The bedside chart and guideline has offered a framework to guide monitoring and management. We did not formally evaluate any change in the confidence of junior doctors managing DKA as part of our audit.

There remain areas for improvement in management with hypoglycaemia rates and potassium replacement. The national DKA survey identified that $67.1 \%$ of patients had a recorded hypoglycaemic event after 24 hours of DKA treatment. ${ }^{3}$ Consideration needs to be given to reducing the rate of intravenous insulin once blood glucose levels have reached $14 \mathrm{mmol} / \mathrm{L}$. This may require review of the monitoring of electrolytes and perhaps management in a HDU setting due to the difficulty in supplementing intravenous potassium safely, as suggested elsewhere. ${ }^{4}$ However, this is difficult to resolve due to bed pressures with high dependency and intensive care units under pressure and

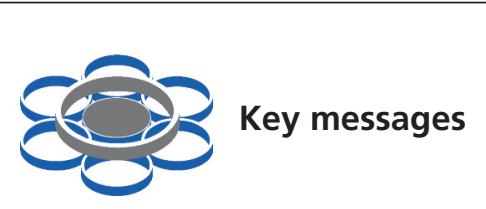

- Diabetic ketoacidosis is a medical emergency and should be treated according to the new JBDS IP Group guidelines when all three diagnostic criteria are met

- There should be a high index of suspicion for diabetic ketoacidosis in any person with diabetes presenting with vomiting

- The new guidelines using FRIII and the use of point of care Capillary Blood Ketone testing has provided an objective end point for resolution of DKA which in this audit was a median of 12 hours

- Long acting insulin should be continued during an episode of diabetic ketoacidosis

less likely to follow the new guideline nationally. ${ }^{8}$ We have found that our DKA guideline has been used $97 \%$ of the time; where it had initially not been used universally in the intensive care department it has now become established practice.

Rates of monitoring of capillary blood glucose and ketones during the intravenous insulin period is above the national average. We think this is due to the prompts for monitoring of any patient on the DKA bedside chart as well as being managed on designated specialty wards and early involvement of the diabetes inpatient care team. However, our potassium monitoring is below standard. In part, we feel that this may be due to the lack of simple nurse-led bedside measures to assess this. Processing tasks done by nursing staff in the first hour tend to be done well, ${ }^{4}$ but tasks that require doctors are often not completed in a timely manner (especially out of hours) due to time constraints.

Median time to resolution of ketosis was found to be 12 hours, which is similar to other audits ${ }^{9}$ and our own previous audit. ${ }^{10}$ Dhatariya et al found this to be 18.7 hours, ${ }^{4}$ which may be due to the nature of data collection for the national survey with more difficult cases put forward to highlight learning points and thus causing a selection bias.

Our inpatient diabetes specialist team forms an integral part of ensuring patient safety. Although we are able to demonstrate that all patients receive a review by our specialist team prior to discharge, this does not necessarily occur at the point of admission. In keeping with national data, most reviews by inpatient specialist teams across the country do not occur in the acute phase. ${ }^{4}$ Although there has been an appropriate increase in patients referred to inpatient diabetes services on average nationally, unfortunately this is not matched by an increase in staffing levels with one-third of UK hospitals having no inpatient diabetes team. ${ }^{6}$ Only $35.5 \%$ of patients with diabetes (not limited to DKA) nationwide are reviewed by an inpatient diabetes team, with consultant diabetologists only having $11.9 \%$ of their time dedicated to the care of inpatients. ${ }^{6,11}$ 


\section{Conclusions}

Overall, we feel that the addition of a local bedside chart and guideline based on the JBDS Inpatient Group guidelines has improved the management of DKA. This has been supported by a consultant-led diabetes inpatient care team which was established in 2012. This has enabled proactive identification of patients admitted to hospital with a diabetes-related emergency earlier in the admission process.

Newer events of inpatient DKA due to insulin omission or a relative lack of insulin in an unwell patient are unfortunately still happening nationally. ${ }^{4}$ This is an area of concern and ongoing work to reduce and minimise this risk. Admitting teams also need to be made aware that subcutaneous insulin administration can be used temporarily, until intravenous access can be established, in situations where intravenous access is difficult.

We have presented data available from before and after implementation of the DKA protocol spanning a 3-year period and auditing 96 patients in total. We have shown that clinically significant improvements have been made in the total time needed on intravenous insulin and, as a consequence, a reduction in associated complications. Rates of hypokalaemia and hypoglycaemia, although improved from pre-protocol, remain suboptimal and further work needs to be done on the transition from fixed rate insulin to a variable rate regimen as well as bedside monitoring of potassium.

\section{Conflict of interest None Funding None}

\section{References}

1. Dreschfeld J. The Bradshawe lecture on diabetic coma. BMJ 1886;2:35863. http://dx.doi.org/10.1136/bmj.2.1338.358

2. Wright J, Ruck K, Rabbitts R, et al. Diabetic ketoacidosis (DKA) in Birmingham, UK, 2000-2009: an evolution of risk factors for recurrence and mortality. Br J Diabetes Vasc Dis 2009;9:278-82. http://dx.doi.org/10.1177/1474651409353248

3. Joint British Diabetes Societies Inpatient Care Group. The Management of Diabetic Ketoacidosis in Adults 2010. http://www. diabetologistsabcd.org.uk

4. Dhatariya KK, Nunney I, Higgins K, et al. National survey of the management of diabetic ketoacidosis (DKA) in the UK in 2014. Diabet Med 2016;33:252-60. http://dx.doi.org/10.1111/dme.12875

5. Health and Social Care Information Centre. National Diabetes Inpatient Audit 2016, 22. http://www.content.digital.nhs.uk/catalogue/PUB23539 (accessed 20 April 2017).

6. Health and Social Care Information Centre. National Diabetes Inpatient Audit 2015, 21 http://www.hscic.gov.uk/catalogue/PUB13662 (accessed 5 Jan 2017).

7. Smith CJ, George GT, Warriner D, et al. Differences in level of confidence in diabetes care between different groups of trainees: the TOPDOC diabetes study. BMC Med Educ 2014;14:191. http://dx.doi.org/10.1186/1472-6920-14-191

8. Rudd B, Patel K, Levy N, et al. A survey of the implementation of the NHS diabetes guidelines for management of diabetic ketoacidosis in the intensive care units of the East of England. JICS 2013;14:60-4 https://doi.org/10.1177/175114371301400112

9. Crasto W, Htike ZZ, Turner L, et al. Management of diabetic ketoacidosis following implementation of the JBDS guidelines: where are we and where should we go? $\mathrm{Br}$ J Diabetes Vasc Dis 2015;15:11-16. http://dx.doi.org/10.15277/bjdvd.2014.040

10. Kurdi $\mathrm{H}$, Pinto LP, Smeeton FJ. An audit of the management of diabetic ketoacidosis in a district general hospital after introduction of the Joint British Diabetes Societies guidelines. Diabet Med 2014;31(Suppl 1): 28-183.

11. Health and Social Care Information Centre. National Diabetes Inpatient Audit 2013, 10. http://content.digital.nhs.uk/searchcatalogue?productid=20443 (accessed 5 Jan 2017). 